\title{
Multi-objective parameters optimization design of self-excited oscillation pulsed atomizing nozzle
}

\author{
Zhaohui Wang $^{1,2} \cdot$ Xiao Sun $^{1,2} \cdot$ Si Chen $^{1,2}$
}

Received: 18 January 2019 / Accepted: 22 September 2019 / Published online: 25 October 2019

(c) The Author(s) 2019

\begin{abstract}
The self-excited oscillation pulsed atomizing nozzle can effectively and evenly spray the high-speed solid cone jet without any extra power. The primary atomization of the jet at the outlet of nozzle directly affects the final spray quality, and the turbulence and cavitation at the outlet of the atomizing nozzle are the other two main factors affecting the atomization. In this work, multi-objective optimization depending on nozzle parameters was established by using mathematical optimization techniques and computational fluid dynamics to improve the jet atomization quality at the outlet of the nozzle. The central composite design method and the response surface method were used to obtain the approximate mathematical model of the primary atomization quality of the jet at the outlet of nozzle. Finally, the non-dominated sorting genetic algorithm with elitist strategy (NSGA-II) and the grey theory were used in combination to optimize the nozzle parameters. Through combining with the NSGA-II and the grey theory, the nozzle parameters were optimized in order to obtain the best primary atomization at the outlet area of nozzle. The optimization results verified the nozzle design with multi-objective optimization method. The optimized values of the turbulent kinetic energy $F_{1}$ and the vapor volume fraction $F_{2}$ increased by $28.26 \%$ and $5.56 \%$, respectively, and the corresponding nozzle parameters of the chamber diameter $D$, the lower nozzle diameter $d_{2}$ and the upper nozzle inlet pressure $P_{\text {in }}$ were, respectively, optimized to $28.056 \mathrm{~mm}, 5.472 \mathrm{~mm}$ and 3.999 Mpa.
\end{abstract}

Keywords Self-excited oscillation pulsed atomizing nozzle $\cdot$ Primary atomization $\cdot$ Multi-objective optimization $\cdot$ Modified cavitation model

\section{Introduction}

Liquid atomization is a physical process in which the liquid is driven by the additional energy to form atomizing droplets in the gas environment. Atomization technology is applied in almost all areas such as industrial manufacturing, agricultural production and daily life, in addition to all kinds of fuel combustion. Atomization technology is also widely used in the combustion fields such as spraying, dusting,

Technical Editor: Daniel Onofre de Almeida Cruz, D.Sc.

Si Chen

1515225801@qq.com

1 Key Laboratory of Metallurgical Equipment and Control of Education Ministry, Wuhan University of Science and Technology, Wuhan, Hubei, China

2 Hubei Key Laboratory of Mechanical Transmission and Manufacturing Engineering, Wuhan University of Science and Technology, Wuhan, Hubei, China humidification and shower. The liquid atomization technology is closely related to industrial production; therefore, it plays an important role in many industrial sectors. However, new challenges shall be posed. On the one hand, increasing the injection pressure can improve the aerodynamic effect of the jet and enhance the spray performances. However, higher injection pressure also increases the energy consumption [1]. On the other hand, the jet shape obtained by the general pressure atomization method is hollow and results in uneven spray and large size of atomized droplets, which is harmful to the quality of spray jet [2]. Therefore, it is urgent to develop a new mechanism and liquid atomization technology to realize the uniform spray without extra energy consumption and break through these two bottlenecks.

The self-excited oscillation pulsed cavitation nozzle is a rotor with a hollow structure. The special nozzle structure and specific boundary conditions generate the self-exited oscillation pulse and transform the continuous jet to the pulsed jet by increasing the pressure peak at the nozzle outlet without the external energy, which improve the spray quality 
$[3,4]$. Using the pulsed jet with self-excited oscillation to improve the atomizing quality is a new spray technology that can effectively address the challenges of hollow jet and uneven spray [5-7]. Therefore, the use of self-excited oscillation pulsed jet technology on liquid atomization and the optimization for qualifying the design the atomizing nozzle possess an exciting prospect for development.

A number of scholars studied the influences of the geometrical and operation parameters on atomization performances by experiments and numerical simulations. Wang et al. [8] established a spray model coupled with nozzle cavitating flow to discover the influence of diesel nozzle geometric parameters including the ratio of nozzle hole length to diameter, entrance curvature radius of nozzle hole and sac volume structure on spray characteristics, which were significant parameters for the structure optimization of the fuel system. Shervani-Tabar et al. [9] studied the influences of injection pressure on the atomizing performances like the spray penetration, the Sauter mean diameter (SMD) and the evaporation of the fuel via numerical simulation. He pointed out the spray penetration and the spray atomization quality would improve with the increase in injection pressure, though SMD would decrease. Park et al. [10] investigated the effects of various design parameters of the twin-fluid nozzle used in wafer cleaning, such as the liquid nozzle diameter, gas slit angle and the number of nozzles on the spray behavior in the experiment. Alexandr et al. [11] studied the effects of the number of tangential passages on the spray cone angle, mass distribution and propellant mixture ratio of a bipropellant atomizer, and three atomizers were designed, manufactured and evaluated experimentally. The results showed that number of tangential passages affects more on the circumferential mass distribution. Yao et al. [12] adopted the single-hole cylindrical injectors with different orifice diameters and lengths to analyze the influences of the nozzle geometry on the distribution of the spray droplet size and corresponding combustion characteristics. They provided important parameters for injector manufacture although lots of time and cost will be spent on those experiments and simulations due to insufficient samples for accuracy improvement. Therefore, simply using experiments or simulation means to optimize the parameters of the selfoscillating pulse (SOP) atomizing nozzle hardly improves the primary atomization quality of the nozzle jet. It is necessary to find another method to optimize the nozzle.

Considering the technology of self-excited oscillation pulsed cavitation jet nozzle, the atomization process can be divided into the primary atomization and the secondary atomization. The primary atomization is the first disintegration of the coherent liquid into big droplets and ligaments near the outlet of nozzle, which strongly depends on the flow conditions inside the injection holes and the starting conditions for the spray breakup [13]. Tamaki et al. [14] and
Geng et al. [15] pointed out that the cavitation effect and turbulence effect are the incentive of the primary atomization of the jet near the nozzle. Therefore, the optimization of the primary atomization quality near the nozzle can be transformed into an optimization issue on the cavitation effect and the turbulent effect at the outlet of nozzle, which is obviously a multi-objective optimization problem.

At present, the computational fluid dynamics (CFD)based multi-objective parameters optimization has been widely used. Uebel et al. [16] used a CFD-based optimization of a quench conversion concept to improve the system performances and obtained the feasible design parameters for the quench reactor. Tahara et al. [17] developed a CFDbased multi-objective parameters optimization for ship design on the optimizer modules of a computer-assisted design (CAD) and CFD. And the multi-objective optimization results appear to be meaningful and prospective. Manshadi et al. [18] used CFD to optimize the three-dimensional wing defined by four design variables and improved approximately aerodynamic efficiency by $15 \%$. Darvish et al. [19] studied the multi-objective optimization of airflow within a ribbed channel to achieve the maximum heat transfer and minimum pressure drop. Through using CFD simulations in conjunction with the neural network and the NSGA-II optimization, valuable and useful results are obtained. Actually, it is hard to get the satisfying results before if no use of the combined method. Paul et al. [20] present the multiobjective optimization on ratio analysis (MOORA) method coupled with the principal component analysis (PCA) in order to achieve the optimal combination of EDM parameters. Safikhani et al. [9] performed a multi-objective optimization on $\mathrm{Al}_{2} \mathrm{O}_{3}$-water nanofluid parameters in flat tubes using CFD techniques, artificial neural networks (ANN) and NSGA-II. It was shown that the obtained Pareto-optimal solutions are valuable for the nanofluid parameter design in flat tubes. In this paper, the self-excited oscillation pulsed cavitation jet nozzle will be optimized as the objective to improve the primary atomization quality at the outlet of the nozzle. Since the optimization of the primary atomization quality is a problem of multi-objective optimization and lots of time and cost shall be taken into account by using experiments and simulations, the CFD-based multi-objective parameter optimization will be an effective method for solving this kind of primary atomization optimization problems.

The self-excited oscillation pulsed cavitation nozzle makes use of their structural characteristics and specific boundary conditions of the self-excited oscillation pulse and consequently produces the cavitation effect and the solid cavitation jet with high-speed pulse to achieve excellent spraying quality. The performances of the self-excited oscillation pulsed atomization nozzle is imperfect, especially near the nozzle area of atomization jet. Meanwhile, jet atomization is the basis of the secondary atomization, 
and the quality of jet atomization will directly affect the jet spray characteristics. Therefore, this article mainly focuses on the jet primary atomization region close to the nozzle for optimization. Turbulence and cavitation are the two main causes resulting in the primary jet atomization that happens in the area close to the self-exited oscillation pulsed nozzle. By optimizing the nozzle parameters, the effects of these two factors on better primary atomization quality will increase. Based on this idea, this paper proposes an approach based on the mathematical optimization and CFD methods for designing the parameters of the self-excited oscillation pulsed atomizer. Aiming at the optimal objectives of nozzle outlet turbulence and cavitation, the jet atomization quality in the area near the jet nozzle is improved firstly.

\section{Numerical calculation model}

\subsection{Geometric model}

The working principle of the self-excited oscillation pulsed cavitation jet nozzle is shown in Fig. 1. Due to the influence of the structure of the self-excited oscillation chamber, periodic vortices form inside the chamber [21]. At the same time, lots of cavitation bubbles are produced at the lower nozzle outlet flow path due to the strong shear between the high-speed jet and the wall of the lower nozzle. Because the high-speed outlet jet has greater turbulence effect and the strong cavitation effect on the outlet of nozzle, it is obvious that the jet near the self-excited oscillation pulsed cavitation jet nozzle will have good primary atomization quality.

The structure of the self-excited oscillation pulsed cavitation jet nozzle includes the upper nozzle, the lower nozzle, the self-excited oscillation chamber and the collision wall as shown in Fig. 2, where $d_{1}$ is the upper nozzle diameter, $d_{2}$ is the lower nozzle diameter, $D$ is the chamber diameter, and $L$ is the chamber length.

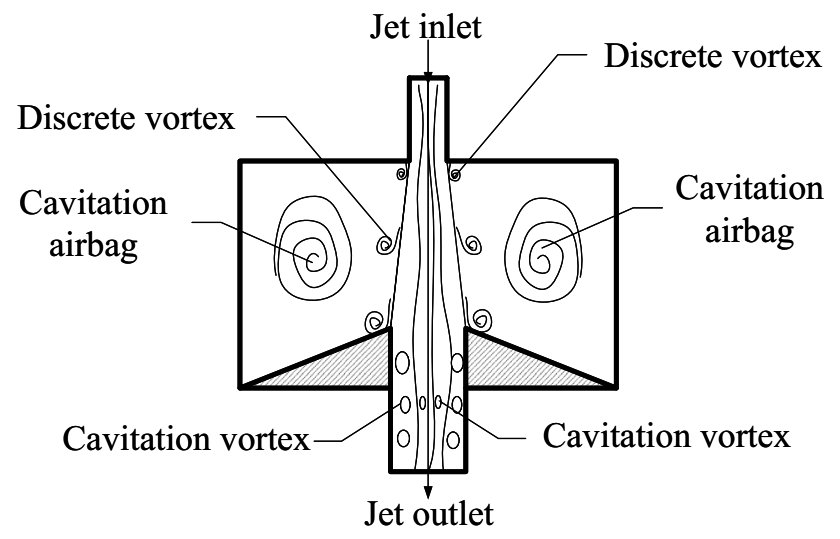

Fig. 1 The working principle diagram

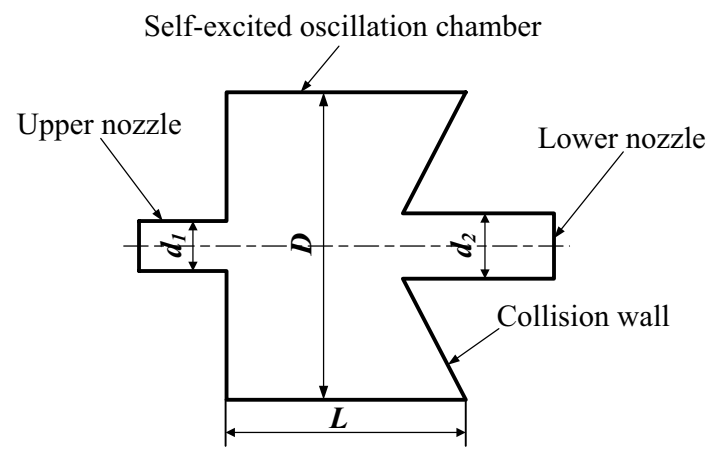

Fig. 2 The structure schematic diagram

\subsection{Cavitation model}

Schnerr-Sauer cavitation model [21] is adopted for simulating the cavitation in the jet nozzle. The net mass transfer equation is

$R=\frac{\rho_{v} \rho_{l}}{\rho} \alpha_{v}\left(1-\alpha_{v}\right) \frac{3}{R_{\mathrm{B}}} \sqrt{\frac{2}{3} \frac{\left(P_{\mathrm{v}}-P\right)}{\rho_{l}}}$

$R_{\mathrm{B}}=\left(\frac{\alpha_{v}}{1-\alpha_{v}} \frac{3}{4 \pi} \frac{1}{n_{B}}\right)^{\frac{1}{3}}$

where $\alpha_{v}$ is the vapor volume fraction, $R_{\mathrm{B}}$ is the bubble radius, $P$ is the local far-field pressure, $P_{\mathrm{v}}$ is the local saturation vapor pressure, $\rho_{v}$ is the vapor phase density, $\rho_{l}$ is the liquid phase density, $\rho$ is density, and $n_{\mathrm{B}}$ is bubble number density.

Sum of shear stress in flow field is

$\tau^{\prime}=\frac{\sqrt{2}}{2}\left(\mu_{m}+\mu_{t}\right)\left(\frac{\partial u}{\partial y}+\frac{\partial v}{\partial x}\right)$

where $\mu_{m}$ is the dynamic viscosity of the mixture media and $\mu_{t}$ is the turbulent viscosity, and $\partial u / \partial y$ is velocity gradient in the direction and $\partial v / \partial x$ is velocity gradient in the direction $Y$.

The local saturated vapor pressure is

$P_{\mathrm{v}}^{\prime}=P_{\mathrm{v}}+0.195 \rho k$

in which $k$ is turbulent kinetic energy and $P_{\mathrm{v}}^{\prime}$ is the local actual saturation vapor pressure.

Considering the cavitation in the jet nozzle is not only related to the local saturated vapor pressure, but also related to the shear stress as well as the pressure pulsations induced by the turbulent kinetic energy. The cavitation model in the nozzle was proposed by Wang $\mathrm{Z}$ et al. [6] as follows: 
When $P \leq P_{\mathrm{v}}^{\prime}+\tau^{\prime}$, the dynamic criterion for cavitation inception is

$$
\begin{aligned}
R_{e} & =\frac{\rho_{v} \rho_{l}}{\rho} \alpha_{v}\left(1-\alpha_{v}\right) \frac{3}{R_{\mathrm{B}}} \\
& \times \sqrt{\frac{2}{3} \frac{\left(P_{\mathrm{v}}+0.195 \rho k+\frac{\sqrt{2}}{2}\left(\mu_{m}+\mu_{t}\right)\left(\frac{\partial u}{\partial y}+\frac{\partial v}{\partial x}\right)-P\right)}{\rho_{l}}}
\end{aligned}
$$

At this point, the cavitation occurs, and the cavitation pressure threshold is $P_{\text {th }}=\operatorname{Min}\left(P_{\mathrm{v}}^{\prime}+\tau^{\prime}\right)$. The cavitation model is established in the Fluent to define the vapor pressure property through the user-defined function (UDF).

\subsection{Turbulence model}

The large eddy simulation (LES) is usually used as the turbulence model for simulating the turbulent flow in the jet nozzle. However, a sufficiently fine mesh especially near the solid wall will be required to adopt the large eddy simulation, which will lead to high computational costs [22]. In recent years, combining with a ReynoldsAveraged Navier-Stokes (RANS) equation solver considering the effect of turbulence, some compromise methods called hybrid RANS/LES are presented to balance the computational cost and accuracy. Therefore, the hybrid RANS/LES method is adopted as the turbulence model in the paper.

In general, the hybrid RANS/LES method can be implemented depending on the RANS turbulence model. The standard k- $\varepsilon$ turbulence model is widely used, and its form is simple. Therefore, in this paper the hybrid RANS/LES method is used on the standard k- $\varepsilon$ turbulence model. The transport equations of the hybrid RANS/LES turbulence model used in this paper are listed as follows [23]:

$\frac{D(\rho k)}{D t}=P-\rho \varepsilon+\frac{\partial}{\partial x_{i}}\left[\left(\mu+\frac{\mu_{t}}{\sigma_{k}}\right) \frac{\partial k}{\partial x_{j}}\right]$

$\frac{D(\rho \varepsilon)}{D t}=C_{\varepsilon 1} \frac{\varepsilon}{k} \cdot P-C_{\varepsilon 2} \rho \frac{\varepsilon^{2}}{k}+\frac{\partial}{\partial x_{j}}\left[\left(\mu+\frac{\mu_{t}}{\sigma_{\varepsilon}}\right) \frac{\partial \varepsilon}{\partial x_{j}}\right]$

$\mu_{t}=f \cdot C_{\mu} \rho \frac{k^{2}}{\varepsilon}$

where $\varepsilon$ is the turbulent energy dissipation, $\mu$ and $\mu_{t}$ stand for mean and fluctuating velocity terms, respectively. $P$ refers to the pressure, $\mu_{t}$ is the turbulent viscosity, $\mathrm{f}$ is the called the control parameter, and the recommended value of $\mathrm{f}$ is 0.38 .

\subsection{Numerical methods}

The numerical simulation is performed for solving the cavitation flow in the self-excited oscillation pulsed cavitation jet nozzle by using the finite volume element method of Fluent. Considering the convergence and calculation speed, a second-order upwind method is used for the convective and diffusive terms. The pressure implicit with splitting of operator (PISO) algorithm is employed to solve the coupling problem between the velocity and pressure fields. Boundary conditions are listed as follows: the inlet boundary condition is set as the pressure at inlet; the outlet boundary condition uses the pressure at outlet set to $1 \mathrm{~atm}$. In addition, it is assumed that liquid phase on the wall surface satisfies the no-slip condition and the wall function is used to deal with the near-wall region.

The self-exited oscillation pulsed cavitation jet nozzle adopts structured grids tiled by quad elements for meshing. In order to observe variation of the pipe flow at the outlet and avoid downstream pressure to disturb the upstream pressure and obtain the fully developed turbulent flow in the pipe, the upper and lower nozzles will be appropriately extended. In this paper, quadrilateral flat structured grids are used for mesh generation. The grid structure of the nozzle is shown in Fig. 3.

The structure parameters and operating parameters of the nozzles are listed as follows: the diameter of the upper nozzle is $6 \mathrm{~mm}$, the diameter of the lower nozzle is $10.8 \mathrm{~mm}$, the length of the cavity is $36 \mathrm{~mm}$, and the cavity diameter is $30 \mathrm{~mm}$. The inlet boundary condition adopts pressure inlet set to $2 \mathrm{MPa}$, and the outlet boundary condition adopts outlet pressure set to a standard atmospheric pressure. The other is wall boundary condition. The PRNS turbulence model and the modified Schnerr and Sauer model are used to solve the multi-phase flow. The time step is $0.0001 \mathrm{~s}$, and the number of time steps is 3000 .

For testing the mesh-dependent, the grid independence is verified by changing the outlet velocity with each scale grid. The comparison among three kinds of meshes with different sizes and densities (1309, 5165 and 20370 grid numbers) is made. Figure 4 shows the results of a grid independency (GI) test. As can been from these figures, all of the generated grids pass the GI test for the pressure P, turbulent kinetic

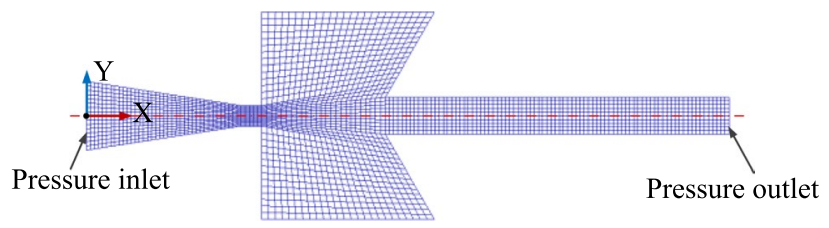

Fig. 3 Grid structure of self-excited oscillation pulsed cavitation jet nozzle 


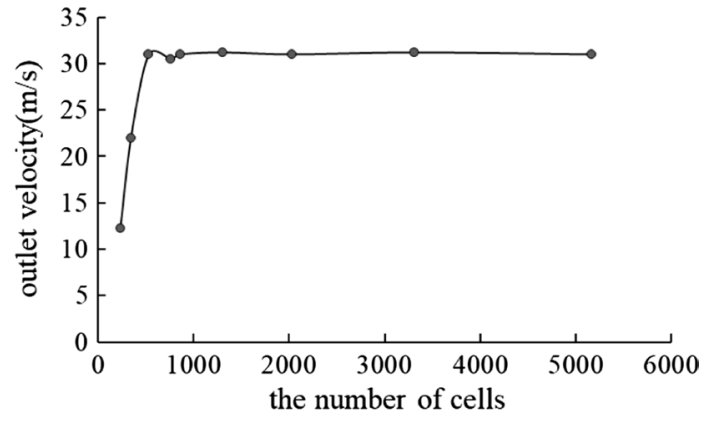

(a) Outlet velocity of each scale grid

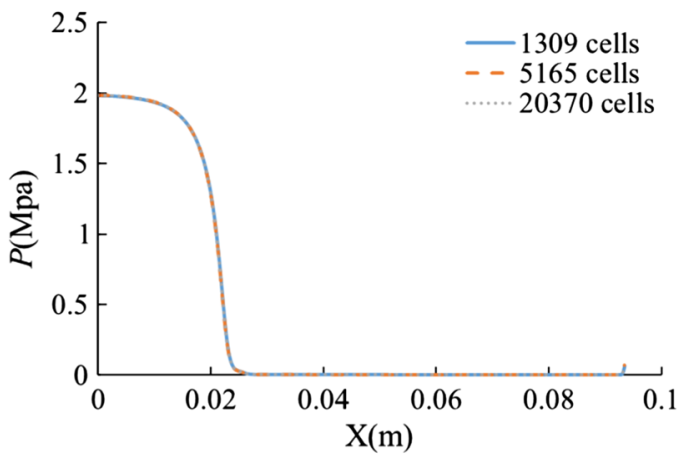

(b) Pressure distribution in X-direction

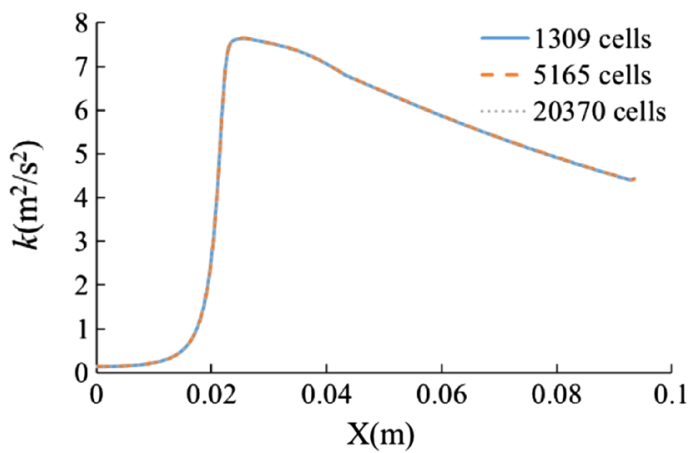

(c) Turbulent kinetic energy distribution in X-direction

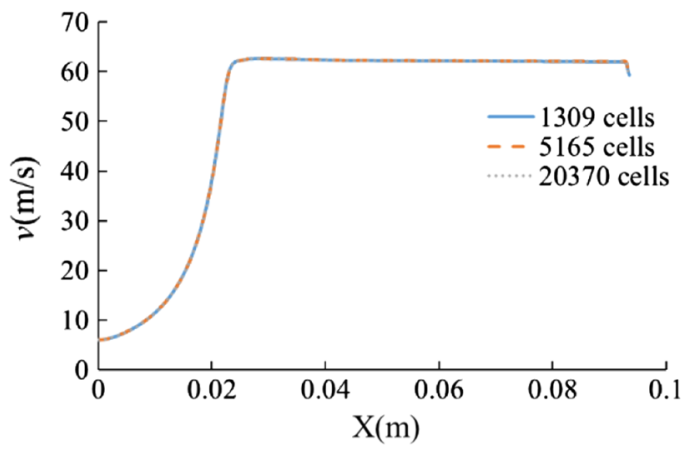

(d) Velocity distribution in X-direction

Fig. 4 Grid independency test

energy $\mathrm{k}$ and velocity v distributed in X-direction. Considering the computing time and cost, the grid number of 1309 is adopted in the simulation.
Table 1 Experimental design factor levels

\begin{tabular}{llll}
\hline Code level & Factor & & \\
\cline { 2 - 4 } & $D /(\mathrm{mm})$ & $d_{2} /(\mathrm{mm})$ & $P_{\text {in }} /(\mathrm{MPa})$ \\
\hline-1 & 28 & 5 & 2 \\
0 & 30 & 5.4 & 3 \\
1 & 32 & 5.8 & 4 \\
\hline
\end{tabular}

\section{Response surface method design}

\subsection{Key design parameters}

The response surface method [24] is used to find the most significant influencing factors. The primary atomization quality is directly affected by structural parameters and operating parameters of the jet nozzle. The structural parameters mainly include the upper nozzle diameter $d_{1}$, the lower nozzle diameter $d_{2}$, the chamber diameter $D$ and the chamber length $\mathrm{L}$. And the operating parameters mainly refer to the upper nozzle inlet pressure $P_{\text {in }}$. The size of the oscillation chamber can be determined by the ratio of the chamber diameter $D$ and the chamber length L. Generally, the upper nozzle diameter $d_{1}$ will be the reference parameter for the other parameters. Therefore, in this paper, the chamber diameter $\mathrm{D}$, the lower nozzle diameter $d_{2}$ and the inlet pressure $P_{\text {in }}$ have to be selected as the design variables for the multi-objective optimization design. Each design variable takes three levels as shown in Table 1.

\subsection{Evaluation indexes for the primary atomization quality}

Since the cavitation effect and turbulence effect are the incentive of the primary atomization of the jet near the nozzle. The study on the optimization of the primary atomization quality near the nozzle can be transformed into the optimization of the cavitation effect and the turbulent effect at the outlet. In order to better establish the approximate mathematical model of the primary atomization near the nozzle, the area-weighted average is more reasonable than the quality weighted average in uneven distribution. The mathematic model is shown as the equations below. Equations (9), (10), (11) and (12) are the maximum of the area-weighted average of turbulent kinetic energy and vapor volume fraction at the cross section of the outlet nozzle. These can be taken as the quantized indexes for the cavitation effect and turbulence effect.

$k^{\prime}=\frac{1}{A} \int k \mathrm{~d} A=\frac{1}{A} \sum_{i=1}^{n} k_{i}\left|A_{i}\right|$

$F_{1}=k_{\text {max }}^{\prime}$ 
$\alpha_{v}^{\prime}=\frac{1}{A} \int \alpha_{v} \mathrm{~d} A=\frac{1}{A} \sum_{i=1}^{n} \alpha_{v i}\left|A_{i}\right|$

$F_{2}=\alpha_{v}^{\prime}$ max

where $k^{\prime}$ is the area-weighted average of the turbulent kinetic energy and $A$ is the total area of the grids and $k$ is the turbulent kinetic energy and $\mathrm{n}$ is the number of grids and $\mathrm{k}_{\mathrm{i}}$ is the turbulent kinetic energy of each grid and $A_{i}$ is the area of each grid and $k_{\text {max }}^{\prime}$ is the maximum area-weighted average of the turbulent kinetic energy and $\alpha_{v}{ }^{\prime}$ is the area-weighted average of the vapor volume fraction and $\alpha \mathrm{v}$ is the vapor volume fraction and $\alpha_{v i}$ is the vapor volume fraction of each grid and $\alpha v_{\text {max }}^{\prime}$ is the maximum area-weighted average of the vapor volume fraction.

\subsection{Response surface approximation model}

Central composite design is adopted for the experiment. Then, the response variables are obtained by Fluent simulation and normalized. The experimental design and the results after normalization are shown in Table 2.

The experimental results are fitted by using $2 F_{1}$ and quadratic regression model. The regression equations between $F_{1}, F_{2}$ and $\mathrm{D}, d_{2}, P_{\text {in }}$ are, respectively, shown in Eqs. (13) and (14).

$$
\begin{aligned}
F_{1} & =-0.083+0.046 D-0.056 d_{2} \\
& +0.75 P_{\text {in }}+0.082 D d_{2}-0.090 D P_{\text {in }}-0.099 d_{2} P_{\text {in }} \\
F_{2} & =0.27+0.019 D+0.45 d_{2} \\
& +0.50 P_{\text {in }}+0.15 D d_{2}-0.17 D P_{\text {in }} \\
& +0.025 d_{2} P_{\text {in }}+0.036 D^{2}-0.058 d_{2}^{2}-0.28 P_{\text {in }}^{2}
\end{aligned}
$$

The following indicators can accurately evaluate the fitting accuracy of the established response surface model according to the test data [25].

1. Complex correlation coefficient $R^{2}$

$$
\begin{aligned}
& R^{2}=\frac{\mathrm{SS}_{R}}{\mathrm{SS}_{Y}}=1-\frac{\mathrm{SS}_{E}}{\mathrm{SS}_{Y}} \\
& \mathrm{SS}_{Y}=\sum_{i=1}^{n}\left(y_{i}-\bar{y}\right)^{2}=\sum_{i=1}^{n} y_{i}^{2}-n \bar{y}^{2} \\
& \mathrm{SS}_{E}=\sum_{i=1}^{n}\left(y_{i}-\bar{y}\right)^{2}
\end{aligned}
$$

\begin{tabular}{|c|c|c|c|c|c|}
\hline \multirow[t]{2}{*}{ Number } & \multicolumn{3}{|c|}{ Design parameters } & \multicolumn{2}{|c|}{ Response parameters } \\
\hline & $D /(\mathrm{mm})$ & $d_{2} /(\mathrm{mm})$ & $P_{\mathrm{in}} /(\mathrm{MPa})$ & $F_{1}$ & $F_{2}$ \\
\hline 1 & 0 & 0 & 0 & -0.1361 & 0.2875 \\
\hline 2 & 0 & 1 & 0 & -0.1294 & 0.504 \\
\hline 3 & 0 & 0 & 0 & -0.1361 & 0.2875 \\
\hline 4 & 0 & 0 & -1 & -0.7289 & -0.5868 \\
\hline 5 & 1 & -1 & 1 & 0.7241 & -0.3087 \\
\hline 6 & 0 & 0 & 0 & -0.1361 & 0.2875 \\
\hline 7 & -1 & 1 & -1 & -1 & -0.4312 \\
\hline 8 & -1 & -1 & -1 & -0.8909 & -1 \\
\hline 9 & 0 & 0 & 1 & 0.6072 & 0.4892 \\
\hline 10 & 1 & 0 & 0 & 0.1247 & 0.2777 \\
\hline 11 & 1 & -1 & -1 & -0.8501 & -0.971 \\
\hline 12 & 1 & 1 & -1 & -0.5868 & 0.3007 \\
\hline 13 & 0 & 0 & 0 & -0.1361 & 0.2875 \\
\hline 14 & -1 & -1 & 1 & 1 & 0.2157 \\
\hline 15 & -1 & 0 & 0 & -0.1519 & 0.2647 \\
\hline 16 & 0 & 0 & 0 & -0.1361 & 0.2875 \\
\hline 17 & -1 & 1 & 1 & 0.5407 & 1 \\
\hline 18 & 0 & 0 & 0 & -0.1361 & 0.2875 \\
\hline 19 & 1 & 1 & 1 & 0.5479 & 0.9452 \\
\hline 20 & 0 & -1 & 0 & -0.0473 & -0.151 \\
\hline
\end{tabular}

Table 2 Experimental design and results

$\mathrm{SS}_{R}=\mathrm{SS}_{Y}-\mathrm{SS}_{E}$

where $\mathrm{SS}_{R}$ is regressive sum of squares that refers to the unevenness of $y$ due to the regression equation and $\mathrm{SS}_{Y}$ is total square sum, which refers to the unevenness of the observed value $y$ and SSE is the square of the error that refers to the unevenness of $y$ due to random errors degree and $\bar{y}$ is the corresponding mean and $n$ is the number of sample points.

2. $\quad$ Modified complex correlation coefficient $R_{\text {adj }}^{2}$

$R_{\text {adj }}^{2}=1-\frac{\mathrm{SS}_{E /}(n-k-1)}{\mathrm{SS}_{Y /}(n-1)}=1-\left(\frac{n-1}{n-1-k}\right)\left(1-R^{2}\right)$

where $k$ is the optimized number of variables and $n$ is the sample points. Unusually, $R_{\text {adj }}^{2}$ always increases when the model variables increase, since the value of $R_{\text {adj }}^{2}$ will decrease if unnecessary items are added.

The $F_{1}$ and $F_{2}$ response surface regression equations were analyzed for accuracy, as shown in Table 3 . The data show that the complex correlation coefficient $R^{2}$ and the adjusted complex correlation coefficient $R_{\text {adj }}^{2}$ are both close to 1 , which indicates that the fitting accuracy of the response surface function is high. 
Table 3 Mean variance analysis table of response surface regression model

\begin{tabular}{lll}
\hline Parameter & \multicolumn{2}{l}{$\begin{array}{l}\text { Response indica- } \\
\text { tors }\end{array}$} \\
\cline { 2 - 3 } & $F_{1}$ & $F_{2}$ \\
\hline$R^{2}$ & 0.9883 & 0.9893 \\
$R_{\text {adj }}^{2}$ & 0.9829 & 0.9796 \\
\hline
\end{tabular}

\subsection{Calculation results}

The coefficients of R-squared for the regression models of $F_{1}$ and $F_{2}$ are 0.9883 and 0.9893 , respectively, which means the fitting degrees of the two regression models are very high. To intuitively illustrate the changes of the turbulent kinetic energy $F_{1}$ and the vapor volume fraction $F_{2}$ at the outlet with the nozzle chamber diameter $D$, the lower nozzle diameter $d_{2}$ and the inlet pressure $P_{\text {in }}$, the response surface analysis are conducted and the response surface of $F_{1}, F_{2}$ with effects of $D, d_{2}, P_{\text {in }}$ is shown in Fig. 5. The turbulent kinetic energy $F_{1}$ at the outlet rises with the increase in the nozzle chamber diameter $D$ and the inlet pressure $P_{\text {in }}$, although it firstly decreases and then increases with the enlargement of the lower nozzle diameter $d_{2}$; the vapor volume fraction $F_{2}$ at the outlet elevates with the enlargement of the nozzle chamber diameter $D$ and the diameter $d_{2}$ of the lower nozzle, although it firstly rises and then declines with increase of the nozzle inlet pressure $P_{\text {in }}$.

And it can be seen from the analysis of variance Tables 4 and 5: the change of the nozzle inlet pressure $P_{\text {in }}$ has an extremely significant influence on the turbulent kinetic energy $F_{1}$ at the outlet. The interaction of the nozzle chamber diameter $D$ and the lower nozzle diameter $d_{2}$, the interaction of the nozzle chamber diameter $D$ and the nozzle inlet pressure $P_{\text {in }}$ and the interaction of the lower nozzle diameter $d_{2}$ and the nozzle inlet pressure $P_{\text {in }}$ have an extremely significant influence on the turbulent kinetic energy $F_{1}$ at the outlet $(p<0.01)$. The lower nozzle diameter $d_{2}$ and the nozzle inlet pressure $P_{\text {in }}$ have an extremely significant influence on the vapor volume fraction $F_{2}$ at the outlet. The interaction of the nozzle chamber diameter $D$ and the lower nozzle diameter $d_{2}$ and the interaction of the lower nozzle diameter $d_{2}$ and the nozzle inlet pressure $P$ have an extremely significant influence on vapor volume fraction $F_{2}$ at the outlet $(p<0.01)$. And the squared term of the nozzle inlet pressure $P_{\text {in }}$ has a more significant influence on the vapor volume fraction $F_{2}$ at the outlet $(p<0.05)$.

\section{Multi-objective optimization}

\subsection{Multi-objective optimization model}

The multi-objective optimization model of the primary atomization near the jet nozzle is shown in Eq. (20):
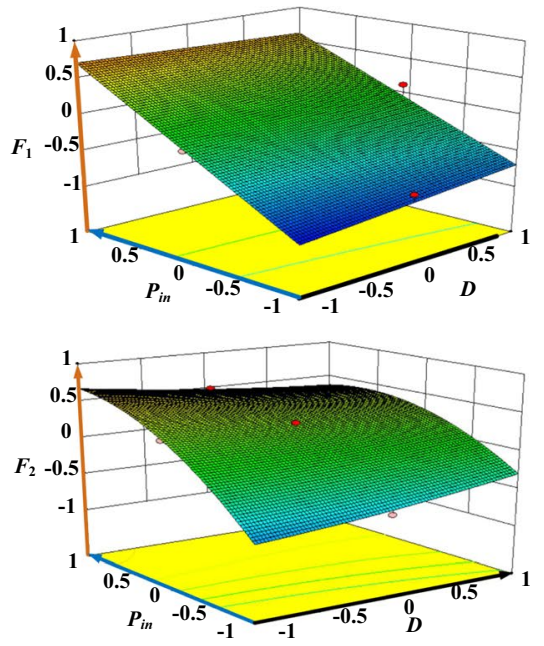

(a) Response surface of $F_{1}, F_{2}$ with combined effect of $P_{i n}, D$
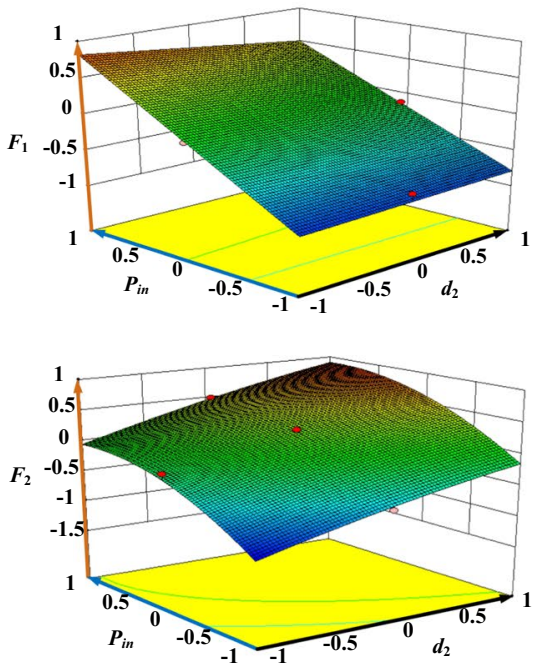

(b) Response surface of $F_{1}, F_{2}$ with combined effect of $P_{i n}, d_{2}$
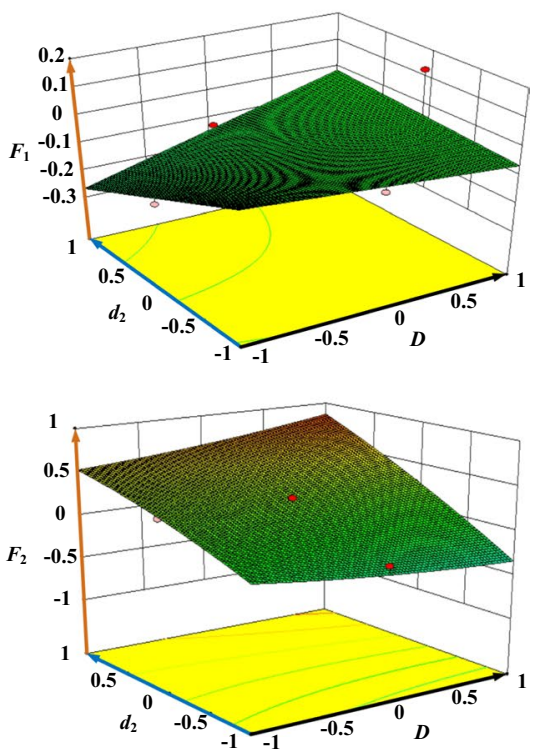

(c) Response surface of $F_{1}, F_{2}$ with combined effect of $d_{2}, D$

Fig. 5 Response surface of $F_{1}, F_{2}$ with combined effect of $D, d_{2}, P_{\text {in }}$ 
Table 4 Analysis of variance in the experimental effect of design parameters on $F_{1}$
Table 5 Analysis of variance in the experimental effect of design parameters on $F_{2}$

\begin{tabular}{llrlrrr}
\hline Source & Sum of squares & $d f$ & Mean square & \multicolumn{1}{l}{ S } & \multicolumn{1}{l}{$p>F$} & Significance \\
\hline Model & 5.84 & 6 & 0.97 & 183.39 & $<0.0001$ & Significant \\
$D$ & 0.021 & 1 & 0.021 & 4.02 & 0.0662 & \\
$d_{2}$ & 0.032 & 1 & 0.032 & 5.98 & 0.0295 & \\
$P_{\text {in }}$ & 5.59 & 1 & 5.59 & 1053.2 & $<0.0001$ & \\
$D d_{2}$ & 0.054 & 1 & 0.054 & 10.12 & 0.0072 & \\
$\mathrm{DP}_{\text {in }}$ & 0.065 & 1 & 0.065 & 12.3 & 0.0039 & \\
$d_{2} P_{\text {in }}$ & 0.078 & 1 & 0.078 & 14.69 & 0.0021 & \\
Residual & 0.069 & 13 & 0.0053 & & & \\
Lack of fit & 0.069 & 8 & 0.0086 & & & \\
Pure error & 0 & 5 & 0 & & & \\
Cor total & 5.91 & 19 & & & &
\end{tabular}

\begin{tabular}{llrlrrr}
\hline Source & Sum of squares & $d f$ & Mean square & \multicolumn{1}{l}{$F$} & \multicolumn{1}{l}{$p>F$} & Significance \\
\hline Model & 5.44 & 9 & 0.6 & 102.34 & $<0.0001$ & Significant \\
$D$ & 0.00379 & 1 & 0.0037 & 0.64 & 0.4417 & \\
$d_{2}$ & 2.06 & 1 & 2.06 & 347.99 & $<0.0001$ & \\
$P_{\text {in }}$ & 2.53 & 1 & 2.53 & 428.29 & $<0.0001$ & \\
$D d_{2}$ & 0.17 & 1 & 0.17 & 29.09 & 0.0003 & \\
$\mathrm{DP}_{\text {in }}$ & 0.22 & 1 & 0.22 & 38.01 & 0.0001 & \\
$d_{2} P_{\text {in }}$ & 0.00488 & 1 & 0.0048 & 0.83 & 0.3845 & \\
$D^{2}$ & 0.0036 & 1 & 0.0036 & 0.62 & 0.4504 & \\
$d_{2}^{2}$ & 0.0093 & 1 & 0.0093 & 1.58 & 0.237 & \\
$P_{\text {in }}^{2}$ & 0.22 & 1 & 0.22 & 37.44 & 0.0001 & \\
Residual & 0.059 & 10 & 0.0059 & & & \\
Lack of fit & 0.059 & 5 & 0.012 & & & \\
Pure error & 0 & 5 & 0 & & & \\
Cor total & 5.5 & 19 & & & &
\end{tabular}

maximum : $F_{1}\left(D, d_{2}, P_{\text {in }}\right)$

maximum : $F_{2}\left(D, d_{2}, P_{\text {in }}\right)$

subject to : $\left\{\begin{array}{l}14 \mathrm{~mm} \leq D \leq 16 \mathrm{~mm} \\ 5 \mathrm{~mm} \leq d_{2} \leq 5.8 \mathrm{~mm} \\ 2 \mathrm{Mpa} \leq P_{\text {in }} \leq 4 \mathrm{Mpa}\end{array}\right.$

In the paper, NSGA-II by Deb for solving multi-objective optimization problem is proposed in order to obtain Pareto-optimal solutions with objective functions $F_{1}$ and $F_{2}$ [26]. The NSGA-II is implemented with an effective sorting method based on non-dominated individual sorting. The method is based on non-dominated personal ranking and crowding distance metric rankings that can assess the overall density of solutions of the same level. It is known that the NSGA-II has a good search performance for widely distributing the Pareto-optimal solutions.
Figure 6 shows all Pareto-optimal solutions obtained by NSGA-II in the multi-objective optimization. As can be seen from the figure, there are conflicts between $F_{1}$ and $F_{2}$. Reducing $F_{1}$ will usually increase $F_{2}$. Due to the same effect of turbulence effect and cavitation effect on the primary atomization quality near the nozzle, it is unable to emphasize on one factor for the optimal solution. Therefore, none of the solutions is absolutely better than the other, and each one of them is an acceptable solution among the Paretooptimal solutions. In order to obtain the optimal primary atomization quality indicated by the optimal solution of $F_{1}$ and $F_{2}$, a compromise method is required.

The grey theory [27] is a new strategy of the multiobjective optimization. The main idea of the grey theory is that when a multi-objective optimization is performed, the optimal solution of each single-objective optimization is as a reference sequence and the sequence is optimized as an objective sequence. The correlation degree between the reference sequence and the objective sequence is generated by using the grey relation of the grey theory. The greater the 


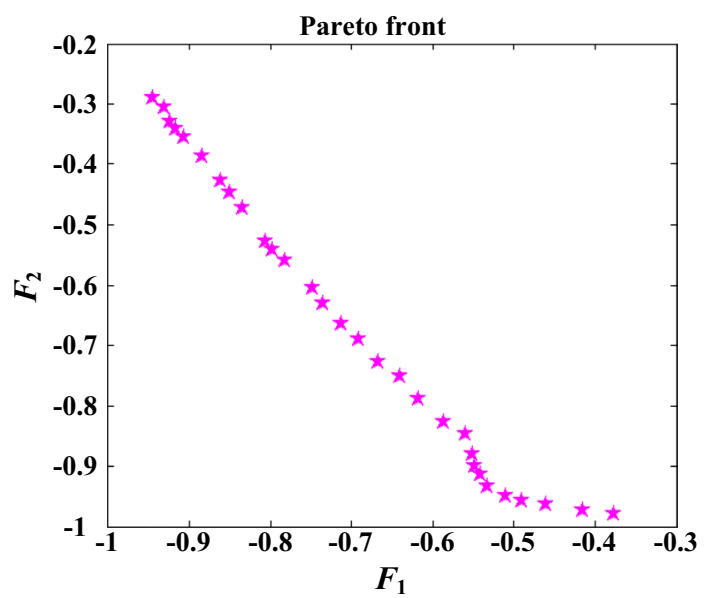

Fig. 6 Pareto-optimal solutions obtained by NSGA-II

correlation degree is, the better the corresponding solution is. Therefore, the multi-objective optimization problem is converted into a single-objective optimization problem. The grey theory is an effective way to solve the optimal solution of the multi-objective optimization problem. In the paper, the grey theory is adopted as the compromising method to seek the optimal solution of $F_{1}$ and $F_{2}$.

The reference sequence is set as $X_{0}=\left\{X_{0}(t), t=1,2, \ldots, n\right\}$. The objective sequence is set as $X_{i}=\left\{X_{i}(t), t=1,2, \ldots n\right\}, i=1,2, \ldots m$, where $m$ is the number of the objective sequences. According to the grey theory, when $t=b$, the grey correlation coefficient [28] of $\mathrm{Xi}$ based on $X_{0}$ is expressed as

$\varepsilon_{0, i}(b)=\frac{\min _{i} \min _{b}\left|x_{0}(b)-x_{i}(b)\right|+\rho \max _{i} \max _{b}\left|x_{0}(b)-x_{i}(b)\right|}{\left|x_{0}(b)-x_{i}(b)\right|+\rho \max _{i} \max _{b}\left|x_{0}(b)-x_{i}(b)\right|}$

where $\rho$ is the resolution coefficient for enhancing the different significance among the correlation coefficients and $0<\rho<1$ and $\rho$ is generally taken as 0.5 .

The correlation degree between the reference sequence and the objective sequence can be calculated by averaging the correlation coefficients which is expressed at each time as

$r_{0, i}=\frac{1}{n} \sum_{b=1}^{n} \varepsilon_{0, i}(b)$

The sequential quadratic programming algorithm is adopted to obtain the optimal values of each single optimization objective function: $F_{1 \max }=42.945$, $F_{2 \max }=0.698 . F_{1 \max }$ and $F_{2 \max }$ are as the reference sequence. The Pareto-optimal solutions obtained by using NSGA-II algorithm are set as the target sequence. According to the grey correlation analysis method, the
Table 6 Correlation data and correlation degree

\begin{tabular}{llll}
\hline Number & Correlation data & Correlation degree \\
\cline { 2 - 3 } & Target sequence & $\begin{array}{l}\text { Reference } \\
\text { sequence }\end{array}$ \\
\hline 1 & & 0.9496 & 0.6894 \\
2 & 0.5102 & 0.8259 & 0.8087 \\
3 & 0.5875 & 0.9001 & 0.7328 \\
4 & 0.5481 & 0.5421 & 0.7814 \\
5 & 0.7974 & 0.4266 & 0.7183 \\
6 & 0.8624 & 0.6644 & 0.9154 \\
7 & 0.7129 & 0.3878 & 0.7022 \\
8 & 0.8849 & 0.8811 & 0.7494 \\
9 & 0.5515 & 0.9643 & 0.6771 \\
10 & 0.4612 & 0.6050 & 0.8446 \\
11 & 0.7481 & 0.3552 & 0.6882 \\
12 & 0.9076 & 0.8456 & 0.7817 \\
13 & 0.5600 & 0.3065 & 0.6751 \\
14 & 0.9309 & 0.7517 & 0.9139 \\
15 & 0.6409 & 0.9785 & 0.6672 \\
16 & 0.3765 & 0.7275 & 0.9659 \\
17 & 0.6673 & 0.3294 & 0.6791 \\
18 & 0.9237 & 0.7895 & 0.8587 \\
19 & 0.6182 & 0.4718 & 0.7407 \\
20 & 0.8353 & 0.5281 & 0.7716 \\
21 & 0.8059 & 0.4464 & 0.7274 \\
22 & 0.8509 & 0.9738 & 0.6702 \\
23 & 0.4150 & 0.6308 & 0.8699 \\
24 & 0.7354 & 0.3412 & 0.6827 \\
25 & 0.9173 & 0.9146 & 0.7199 \\
26 & 0.5419 & 0.9335 & 0.7037 \\
27 & 0.5334 & 0.2910 & 0.6678 \\
30 & 0.9456 & 0.78930 & 0.7975 \\
\hline & 0.6922 & 0.962 \\
29 & 0.4906 & 0.6828 \\
\hline & &
\end{tabular}

correlation degree between the reference sequence and the target sequence is obtained. When the correlation degree is the largest, the solution is the optimal solution for this article. Then, the correlation coefficients are calculated in accordance with Eq. (21). Finally, the correlation degrees of each sequence are calculated by Eq. (22) as shown in Table 6.

\subsection{Results analysis and discussion}

The optimal result is number 16 in Table 6 , and the maximum value of correlation degree is 0.9659 and the corresponding optimal values of the turbulent kinetic energy $F_{1}$ and the vapor phase volume fraction $F_{2}$ are 40.522 and 0.683 . Meanwhile, the corresponding parameters $\mathrm{D}, d_{2}$ and $P_{\text {in }}$ are $28.056,5.472$ and 3.999. The simulated values of 
Fig. 7 Curves of the area-weighted average of turbulent kinetic energy and vapor volume fraction at the outlet nozzle

$F_{1}$ and $F_{2}$ calculated by using the optimal parameters are 40.899 and 0.695 as shown in Fig. 7a. It can be seen that the differences of $F_{1}$ and $F_{2}$, respectively, obtained by the analytical method and the numerical simulation are $0.92 \%$ and $1.72 \%$. The calculated values of $F_{1}$ and $F_{2}$ by these two methods are very close. It illustrates that the mathematical model of the primary atomization near the jet nozzle is correct, which can approximately substitute the simulation method. The values of $F_{1}$ and $F_{2}$ by using the original parameters (30, 5.4 and 3 in Table 1) are 31.593 and 0.647 as shown in Fig. 7b. The optimal values of $F_{1}$ and $F_{2}$ increase by $28.26 \%$ and $5.56 \%$, respectively, compared with the original values. Correspondingly, the values of frequency and amplitude get double. This indicates the energy increases and furthermore the nozzle effect improves.

Furthermore, the distributions of the turbulent kinetic energy and the vapor phase volume fraction on the nozzle exit(outlet) section are shown in Figs. 8 and $9(T=0.3 \mathrm{~s})$. As can be seen from the figures, the turbulent kinetic energy $F_{1}$ and the vapor phase volume fraction $F_{2}$ determined by the optimal parameters are generally higher than the original values on the nozzle outlet section. The above results illustrate that multi-objective optimization for improving the primary atomization quality near the nozzle is feasible.

\section{Conclusions}

The jet primary atomization quality near the nozzle directly affects the final quality of the spray. The nozzle outlet turbulence and cavitation are the principal causes of the primary jet atomization. In addition, the optimization of nozzle parameters is used to improve these two factors for better primary atomization quality. Since some problems, such as the ultra-low accuracy of optimal solution and the much costs for labor and time, still exist in the design of atomization nozzle at present, a design method based on multiobjective optimization technique and CFD is proposed in this paper. Some magnificent conclusions are drawn as the following.

1. Depending on the response surface analysis, the turbulent flow energy $F_{1}$ at the outlet increases with the nozzle chamber diameter $D$ and the inlet pressure $P_{\text {in }}$, though it decreases firstly and then increases with the enlargement of the lower nozzle diameter $d_{2}$; the vapor phase volume fraction at the outlet $F_{2}$ gets higher as the nozzle chamber diameter $D$ and the lower nozzle diameter $d_{2}$ increase, though it increases firstly and then decreases as the nozzle inlet pressure $P_{\text {in }}$ is amplified.
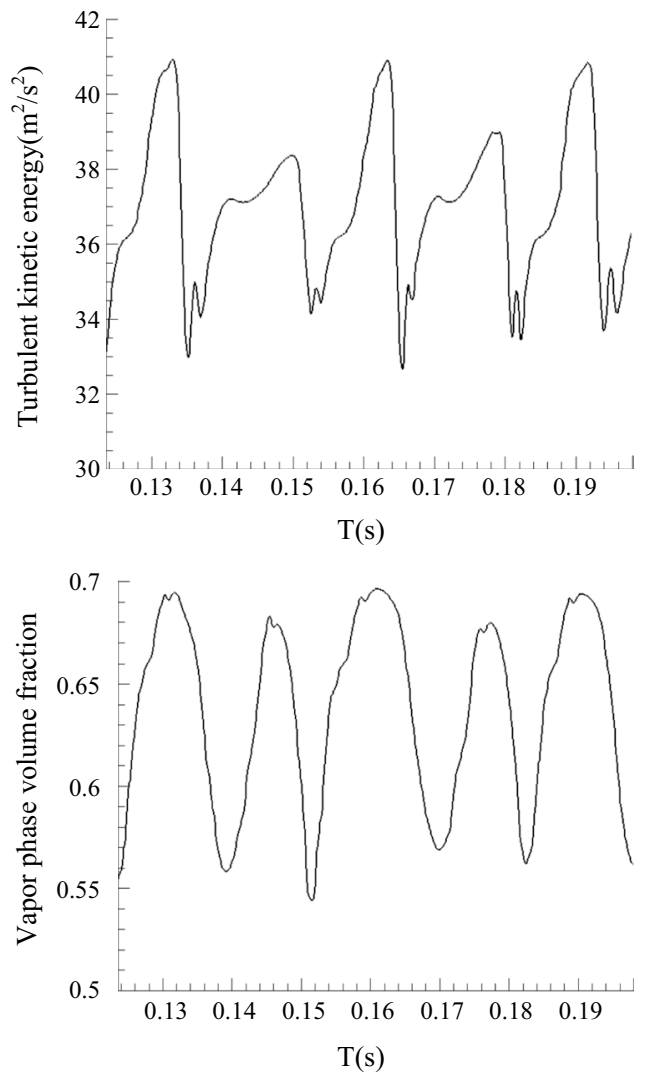

(a) The optimal parameters
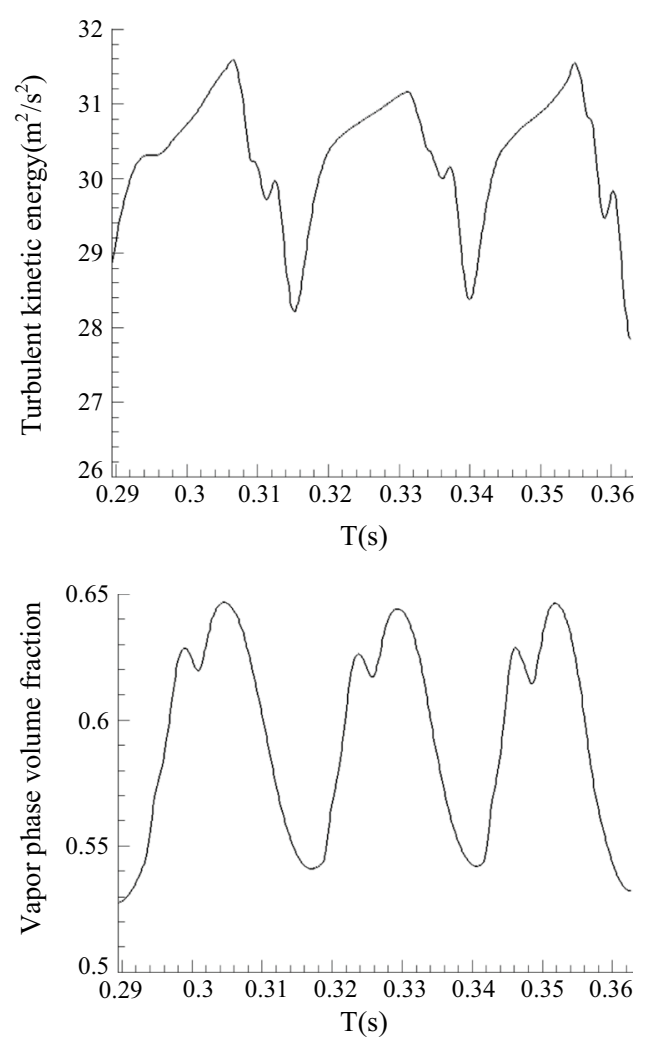

(b) The original parameters 


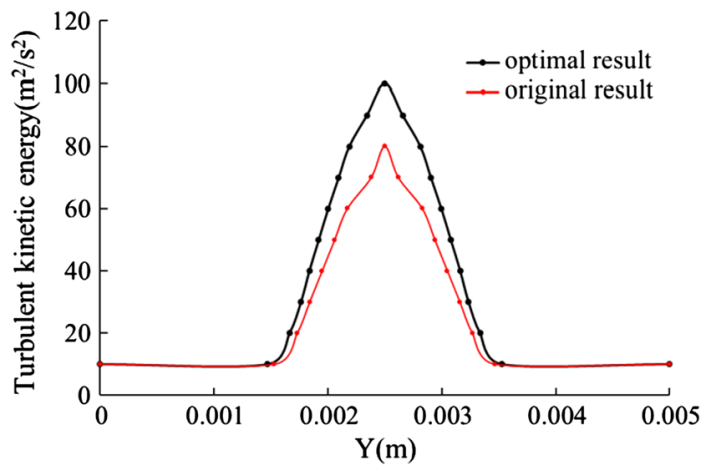

Fig. 8 Distribution of the turbulent kinetic energy on the nozzle outlet section of $Y$-direction

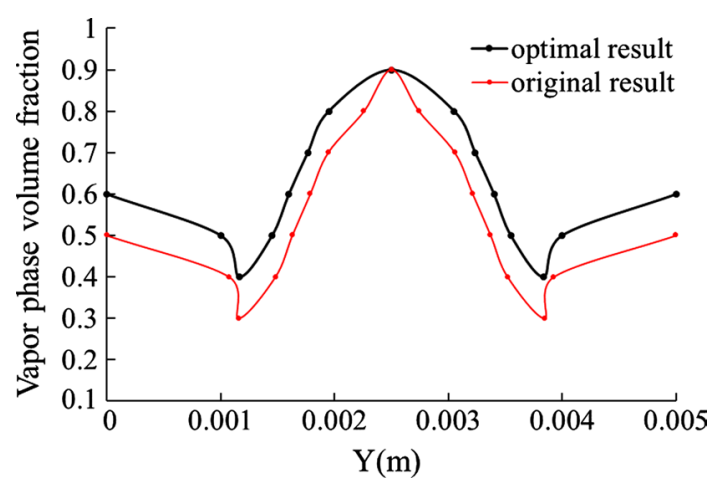

Fig. 9 Distribution of the vapor phase volume fraction on the nozzle outlet section of $Y$-direction

The approximate mathematical model of the primary atomization quality of the jet near the outlet nozzle was established by the response surface method.

2. Using the combined algorithm with NSGA-II and grey theory, the optimized set of nozzle parameters is determined relied on the optimal primary atomization near the nozzle area. The difference between the analytical and the numerical solutions is minor. The atomization model established for the nozzle is correct; therefore, the model can approximatively take place of the simulation results.

3. It is feasible to improve the primary atomization quality of the jet flow near the nozzle area by the multi-objective optimization. The optimized values of the turbulent kinetic energy $F_{1}$ and the vapor volume fraction $F_{2}$ increase by $28.26 \%$ and $5.56 \%$. Depending on the analysis of the distribution of turbulent kinetic energy and the vapor phase volume fraction in the nozzle outlet section, the optimized turbulent kinetic energy and the vapor phase volume fraction in the nozzle outlet section are generally higher than the original values.
Acknowledgements This work was supported by the National Natural Science Foundation of China under Grant Nos. 51875419 and 51405352 .

Open Access This article is distributed under the terms of the Creative Commons Attribution 4.0 International License (http://creativeco mmons.org/licenses/by/4.0/), which permits unrestricted use, distribution, and reproduction in any medium, provided you give appropriate credit to the original author(s) and the source, provide a link to the Creative Commons license, and indicate if changes were made.

\section{References}

1. Yu S, Yin B, Deng W et al (2018) Experimental study on the spray characteristics discharging from elliptical diesel nozzle at typical diesel engine conditions. Fuel 221:28-34. https://doi. org/10.1016/j.fuel.2018.02.090

2. Santolaya JL, Aísa LA, Calvo E et al (2010) Analysis by droplet size classes of the liquid flow structure in a pressure swirl hollow cone spray. Chem Eng Process 49(1):125-131. https://doi. org/10.1016/j.cep.2009.12.003

3. Wang Z, Hu Y, Liao Z, Gao Q, Chen S (2016) Cavitation characteristics study on the outlet channel of atomization nozzle based on the self-excited oscillating pulse effects. J Mech Eng 52(14):204-213. https://doi.org/10.3901/JME.2016.14.204

4. Wang Z, Rao C, Gao Q, Sun X, Wang Y (2018) Study on the pulsation of self-excited oscillation chamber based on instantaneous vortex boosting effect. J Mech Eng 54(14):207-214

5. Wang Z, Hu Y, Chen S, Zhou L, Xu W, Lien F (2019) Investigation of self-excited oscillation chamber cavitation effect with special emphasis on wall shape. Trans Can Soc Mech Eng. https ://doi.org/10.1139/tcsme-2019-0005

6. Wang Z, Chen S, Deng X (2017) Research on modified cavitation model for self-excited oscillation pulsed jet nozzle. Int Conf Mech IEEE. https://doi.org/10.1109/ICMSC.2017.7959449

7. Wang Z, Hi Y, Rao C, Deng X (2017) Numerical analysis of cavitation effects of self-excited oscillation pulse nozzles and jet forms. China Mech Eng 28(13):1535-1541. https://doi. org/10.3969/j.issn.1004-132X.2017.13.004

8. Wang F, He Z, Liu J, Wang Q (2015) Diesel nozzle geometries on spray characteristics with a spray model coupled with nozzle cavitating flow. Int J Automot Technol 16(4):539-549. https://doi. org/10.1007/s12239-015-0055-9

9. Shervani-Tabar MT, Sheykhvazayefi M, Ghorbani M (2013) Numerical study on the effect of the injection pressure on spray penetration length. Appl Math Model 37(14-15):7778-7788. https ://doi.org/10.1016/j.apm.2013.03.002

10. Park J, Kang J, Park S (2016) Comparisons of spray characteristics depending on the nozzle parameters in a wafer cleaning system using a twin-fluid nozzle. J Electrochem Soc 163(3):E88-E96. https://doi.org/10.1149/2.0811603jes

11. Alexandre A, Pedro TL, Cristiane AM (2013) Effects of the number of tangential passages on spray characteristics of a bipropellant atomizer. J Braz Soc Mech Sci Eng 36(3):583-590. https:// doi.org/10.1007/s40430-013-0101-3

12. Yao C, Geng P, Yin Z, Hu J, Chen D, Ju Y (2016) Impacts of nozzle geometry on spray combustion of high pressure common rail injectors in a constant volume combustion chamber. Fuel 179:235-245. https://doi.org/10.1016/j.fuel.2016.03.097

13. Salvador FJ, Ruiz S, Crialesi-Esposito M et al (2018) Analysis on the effects of turbulent inflow conditions on spray primary atomization in the near-field by direct numerical simulation. Int 
J Multiph Flow 102:49-63. https://doi.org/10.1016/j.ijmultipha seflow.2018.01.019

14. Tamaki N, Nishida K, Shimizu M, Hiroyasu H (2001) Increase of the atomization of a liquid jet by cavitation in a nozzle hole. Nihon Kikai Gakkai Ronbunshu B Hen Trans Jpn Soc Mech Eng Part B 63(613):3144-3149

15. Geng L, Wang C, Wei Y, Li Q (2017) Simulation on internal flow characteristics of nozzle for diesel engine fueled with biomass blend fuel. Trans Chin Soc Agric Eng 33(21):70-77. https://doi. org/10.11975/j.issn.1002-6819.2017.21.008

16. Uebel K, Rößger P, Prüfert U, Richter A, Meyer B (2016) CFDbased multi-objective optimization of a quench reactor design. Fuel Process Technol 149:290-304. https://doi.org/10.1016/j. fuproc.2016.04.008

17. Tahara Y, Tohyama S, Katsui T (2010) CFD-based multi-objective optimization method for ship design. Int J Numer Methods Fluids 52(5):499-527. https://doi.org/10.1002/fld.1178

18. Manshadi MD, Aghajanian S (2018) Computational aerodynamic optimization of wing-design concept at supersonic conditions by means of the response surface method. J Braz Soc Mech Sci Eng 40(5):254. https://doi.org/10.1007/s40430-018-1150-4

19. Darvish DM, Safikhani H, Yahyaabadi M (2017) Multi-objective optimization of asymmetric v-shaped ribs in a cooling channel using CFD, artificial neural networks and genetic algorithms. J Braz Soc Mech Sci Eng 39(6):2319-2329. https://doi.org/10.100 7\%2Fs40430-016-0698-0

20. Paul TR, Saha A, Majumder H, Dey V, Dutta P (2019) Multiobjective optimization of some correlated process parameters in EDM of Inconel 800 using a hybrid approach. J Braz Soc Mech Sci Eng 41:300. https://doi.org/10.1007/s40430-019-1805-9

21. Safikhani H, Abbassi A, Khalkhali A, Kalteh M (2014) Multiobjective optimization of nanofluid flow in flat tubes using CFD, artificial neural networks and genetic algorithms. Adv
Powder Technol 25(5):1608-1617. https://doi.org/10.1016/j. apt.2014.05.014

22. Piomelli U, Balaras E (2008) Wall-layer models for largeeddy simulations. Prog Aerosp Sci 44(6):437-446. https://doi. org/10.1016/j.paerosci.2008.06.001

23. Wang B, Zha G (2013) Comparison of a low diffusion E-CUSP and the roe scheme for RANS calculation. Aiaa J. https://doi. org/10.2514/6.2008-569

24. Zhu F, Wang Z, Lv M (2015) Multiobjective optimization of precision forging process parameters based on response surface method. Adv Mater Sci Eng 7:730-893. https://doi. org/10.1155/2015/893730

25. Ai YS (2012) Research on response surface method optimization based on radial basis function. Dissertation, Huazhong University of Science and Technology, Hubei, China

26. Deb K, Agrawal S et al (2000) A fast elitist non-dominated sorting genetic algorithm for multi-objective optimization: NSGAII. Parallel Prob Solv Nat PPSN VI 1917:849-858. https://doi. org/10.1007/3-540-45356-3_83

27. Cai K, Wang D (2017) Optimizing the design of automotive $S$-rail using grey relational analysis coupled with grey entropy measurement to improve crashworthiness. Struct Multidiscip Optim 56(6):1539-1553. https://doi.org/10.1007/s00158-017-1728-y

28. Ramesh K, Jaiganesh V, Malarvannan RRR (2019) Optimization of drilling parameters in hybrid $\left(\mathrm{A} 16061 / \mathrm{SiC} \mathrm{B}_{4} \mathrm{C} / \mathrm{talc}\right)$ composites by grey relational analysis. J Braz Soc Mech Sci Eng 41:3. https://doi.org/10.1007/s40430-019-1661-7

Publisher's Note Springer Nature remains neutral with regard to jurisdictional claims in published maps and institutional affiliations. 